\title{
Effects of Stress and Morphology on the Resistivity and Critical Temperature of Room-Temperature-Sputtered Mo Thin Films
}

\author{
Lourdes Fàbrega, Iván Fernández-Martínez, María Parra-Borderías, Óscar Gil, Agustín Camón, \\ Raquel González-Arrabal, Javier Sesé, José Santiso, José-Luis Costa-Krämer, and Fernando Briones
}

\begin{abstract}
We report on the structural and electrical characterization of Mo thin films deposited at room temperature by RF magnetron sputtering. The effect of RF power on the morphology and residual stress of the films is analyzed. The films are under compressive stress and consist of densely packed columns with a lateral size on the order of $20 \mathrm{~nm}$. The stress, critical temperature, and resistivity of the films are found to rise when increasing the ejected ion energy during the sputtering process. The changes in critical temperature and resistivity are discussed in terms of the observed morphology and stress changes.
\end{abstract} films.

Index Terms-Conductivity, sputtering, stress, superconducting

\section{INTRODUCTION}

$\mathbf{M}$ OLYBDENUM THIN films are of interest in various technological areas [1]-[6]. Because of their remarkable properties (high melting point, high conductivity, good chemical stability, and high hardness), traditionally, they have extensively been used in microelectronics (gates for MOS integrated circuits, interconnections, and diffusion barriers) and engineering and as back contacts for solar cells. More recently, they are considered as the most suitable candidates to fabricate superconducting microcalorimeters for high-performance radiation detectors, the so-called transition-edge sensors (TESs).

As a consequence of their appeal, a variety of deposition techniques and conditions has been employed to obtain Mo

Manuscript received March 17, 2009; revised April 16, 2009. First published September 4, 2009; current version published November 25, 2009. This paper was recommended by Associate Editor N. Newman. This work was supported by the Spanish Ministerio de Ciencia e Innovación (MCINN) under Projects ESP2006-13608-C02 and AYA2008-00591/ESP.

L. Fàbrega and Ó. Gil are with the Institut de Ciència de Materials de Barcelona, Consejo Superior de Investigaciones Científicas (CSIC), 08193 Barcelona, Spain (e-mail: lourdes@icmab.es).

I. Fernández-Martínez, J.-L. Costa-Krämer, and F. Briones are with the Instituto de Microelectrónica de Madrid, CSIC, 28760 Madrid, Spain.

M. Parra-Borderías and A. Camón are with the Instituto de Ciencia de Materiales de Aragón and Departamento de Física de la Materia Condensada, CSIC, Universidad de Zaragoza, 50009 Zaragoza, Spain.

R. González-Arrabal is with the Instituto de Microelectrónica de Madrid, CSIC, 28760 Madrid, Spain, and also with the Instituto de Fusión Nuclear, ETSI, 28006 Madrid, Spain.

J. Sesé is with the Instituto de Nanociencia de Aragón, Universidad de Zaragoza, 50009 Zaragoza, Spain.

J. Santiso is with the Centro de Investigación en Nanociencia y Nanotecnología (CIN2), Institut Català de Nanotecnologia, CSIC, 08193 Barcelona, Spain.

Color versions of one or more of the figures in this paper are available online at http://ieeexplore.ieee.org.

Digital Object Identifier 10.1109/TASC.2009.2027609 thin films [1]-[20]. The most used techniques are sputtering (dc and RF) and electron-beam (e-beam) evaporation. Among all possible deposition configurations, special attention has been devoted to room temperature deposition, which would ease fabrication processes, avoiding mechanical or electrical problems in Mo-based devices. One of the most important properties of Mo thin films to be used for device applications is their state of stress, since it will affect their adherence to the substrate and alter their functional properties. In general, low-stress films have been pursued to achieve better substrate adherence.

The effects of deposition conditions on the stress and morphology of Mo thin films have been rather widely analyzed [7]-[11]. Because of the relevance of the electric conductivity of the films, some studies [2]-[4], [12]-[14] were focused on the correlation of morphology and stress with electric properties - mainly room temperature resistivity. However, even when Mo thin films have been proposed as very suitable candidates for TESs, only a few papers are devoted to the correlation of stress and morphology with the superconducting critical temperature [5], [6], [15]-[18]. In fact, there is a considerable lack of understanding of how morphology and stress affect the superconducting properties of Mo thin films, which hampers further improvement of superconducting Mobased devices.

In this paper, we study the effect of deposition parameters on the morphology, stress, resistivity, and critical temperature of Mo thin films deposited by RF magnetron sputtering at room temperature. It will be shown that raising the bias voltage results in an increase of residual stress, resistivity, and critical temperature of the films. The correlations between these parameters will be analyzed.

\section{EXPERIMENTAL}

Mo thin films with a thickness of $50 \mathrm{~nm}$ were deposited on top of $\mathrm{Si}$ single-crystal substrates covered by a $300-\mathrm{nm} \mathrm{Si}_{3} \mathrm{~N}_{4}$ layer. The deposition was carried out by RF magnetron sputtering at room temperature and in an ultrahigh-vacuum system with a base pressure of $\sim 10^{-7} \mathrm{~Pa}$. The Ar working pressure was kept constant at $0.5 \mathrm{~Pa}$ during sputtering, and the RF power was varied between 25 and $213 \mathrm{~W}$. The distance between the substrate and the target was $8 \mathrm{~cm}$ in all cases. The purity of the target was $99.95 \%$. Prior to deposition, the substrates were cleaned using a $\mathrm{KOH}$ solution (15 wt.\% concentration). 
X-ray diffraction (XRD) was employed to study the crystal structure, preferential orientations, and residual stress of the layers. $\theta-2 \theta$ scans at different angles $\Psi$ between the normal to the film and the normal to the diffraction planes were performed using a Bruker D8 Advance diffractometer with a GADDS 2-D detector. The thickness of the films was determined from reflectometry measurements.

Morphological characterizations were performed by combining different microscopy techniques, namely, transmission electron (TEM), scanning electron (SEM) and atomic force (AFM) microscopies. For TEM, thin foils were prepared in planar view by mechanical flat polishing down to $20 \mu \mathrm{m}$ and final $\mathrm{Ar}^{+}$bombardment at $V=4 \mathrm{kV}$ with an incident angle of $7^{\circ}$ focusing on the sample from above. A Jeol 2100 working at $200 \mathrm{kV}$ was used in bright-field mode. SEM images were taken with a microscope QUANTA FEI 200 FEG-ESEM operated at $10 \mathrm{kV}$ and at a residual back pressure of $2.5 \cdot 10^{-4} \mathrm{~Pa}$ in the operating chamber. AFM images were taken by a commercial (Agilent 4500) microscope operated in tapping mode at a frequency between 146 and $236 \mathrm{kHz}$. $\mathrm{Si}_{3} \mathrm{~N}_{4}$ tips of 2- and 20-nm diameters were used. The topography was analyzed by the software Mountains from Digital Surf.

The chemical composition of the films was investigated by means of Rutherford backscattering spectrometry (RBS). RBS measurements were done using a He beam at an energy of 2.0 MeV in a chamber, which is connected to a 5-MV Tandetron accelerator [21], [22] at the Center for Microanalysis of Materials, Universidad Autónoma de Madrid. The backscattered ions were detected by a standard Si-barrier detector (ORTEC BU012-050-500) located at an angle of $170^{\circ}$. The chemical composition of the samples was estimated by comparing experimental and simulated RBS spectra. For the simulations, the SIMNRA commercial computer code [23] was used.

The temperature dependence of the resistance was measured in a commercial Physical Property Measurement System from Quantum Design, using the four-point resistance method. $T_{c}$ was defined as the point at the middle of the resistive transition (50\% of the residual resistance value).

\section{RESUltS AND Discussion}

When thin films are deposited by RF magnetron sputtering, a change in the RF power $W$ has always associated a change in the dc bias $V_{c}$ and, therefore, on the deposition rate $G_{r}$. As shown in Fig. 1, these three parameters, namely, $W, V_{c}$, and $G_{r}$, can be considered to be nearly proportional for the studied pressure range. In our particular case, the deposition rate varies from 0.1 to $0.7 \mathrm{~nm} / \mathrm{s}$ by increasing $V_{c}$ from 250 to $960 \mathrm{~V}$. The changes of growth dynamics due to variations of both the rate of arriving atoms and their energy are expected to affect the stress, defect structure, and morphology of the films. Because the aforementioned three parameters, which characterize growth, differ only by a factor in our experiment, the following analyses and discussion will be carried out using only one of them as a variable. Since for a fixed pressure and target-substrate distance, the initial energy of the atoms ejected from the target is mainly determined by $V_{c}$, and because we have obtained more reproducible films when

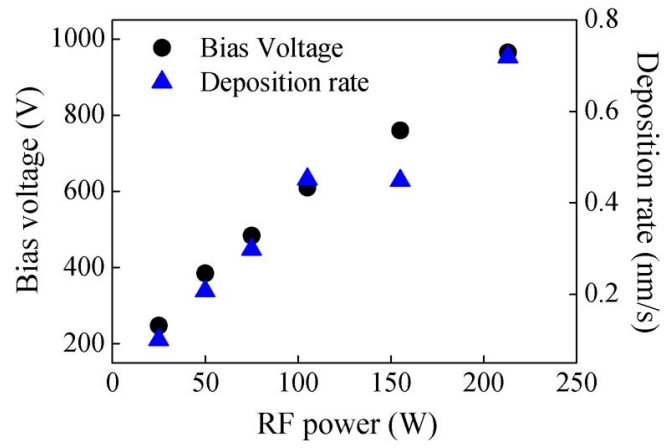

Fig. 1. Bias voltage and deposition rate as a function of the RF power to the target for the studied films. A linear relationship between the three parameters is observed.

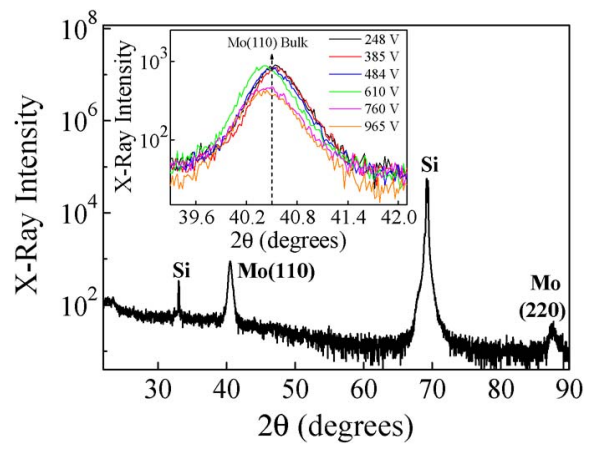

Fig. 2. XRD $(\theta-2 \theta$ scan $)$ for the film deposited at 248 V. Only the Si peaks and the $(h h 0)$ reflections of Mo are appreciated, indicating a preferential orientation along this direction. This scan is representative of all the films. (Inset) Detail of the evolution of the (110) peak position as a function of the bias voltage $V_{c}$. The dashed line corresponds to that of bulk Mo.

fixing its value during the deposition process, we decide to choose this parameter to be the one determining the deposition procedure.

\section{A. Structure and Morphology}

XRD patterns for films deposited at different $V_{c}$ are shown in Fig. 2. Only the (110) reflection of Mo is seen, indicating that all the films are single phase and display a preferential orientation along the (110) direction. The intensity of the main (110) peak does not display any relevant dependence on $V_{c}$. Nevertheless, an increase in the peak width and a shift in its position toward lower angles are observed with increasing $V_{c}$. These data point to the existence of some residual stress (lattice expansion along the growth direction).

The residual stress has been more carefully analyzed by the so-called $\sin ^{2} \Psi$ technique [24], consisting of measuring $\theta-2 \theta$ scans at different $\Psi$ angles. Monitoring of the interplanar distance $d$ for a certain reflection (obtained from the $\theta-2 \theta$ peak position at a given $\Psi$ ) as a function of $\sin ^{2} \Psi$ provides information on the stress of the film. All investigated films show a linear dependence of $d$ on $\sin ^{2} \Psi$, with a negative slope. The linear dependence indicates that the films exhibit a biaxial stress in-plane, whereas the negative slope reveals that this in-plane stress is compressive, in agreement with the tensile out-of-plane stress revealed by standard $\theta-2 \theta$ scans (Fig. 2). 


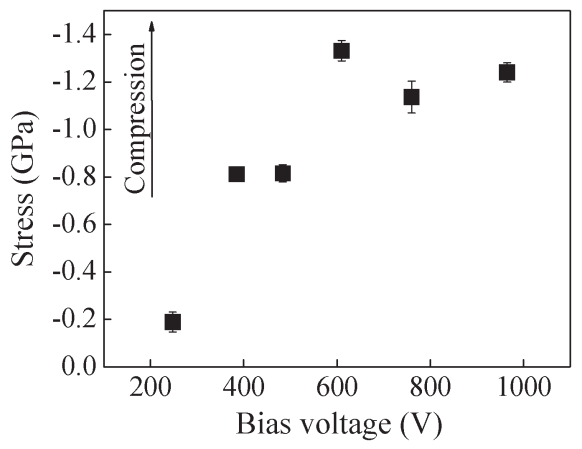

Fig. 3. Residual stress of the films as a function of the bias voltage $V_{c}$. Note that the vertical axis is reversed to emphasize that the compressive stress increases with $V_{c}$.

The residual stress of the films is directly proportional to the absolute value of the slope of $d$ versus $\sin ^{2} \Psi$; using these slopes and tabulated elastic constants for Mo $(E=320 \mathrm{GPa}$ and $\nu=0.32$ [7]), we have estimated the residual stress values as a function of $V_{c}$. They are displayed in Fig. 3, which reveals a clear enhancement of stress when increasing the dc bias from $-190 \mathrm{MPa}$ (for $V_{c}=248 \mathrm{~V}$ ) to $-1.3 \mathrm{GPa}$ (for $V_{c}=$ $610 \mathrm{~V})$.

The values in Fig. 3 agree with those reported in other works [6], [7], [9], [11], [17], [25], which showed that stress in sputtered Mo films can be tuned from $-1.4 \mathrm{GPa}$ to $+1 \mathrm{GPa}$ by changing the deposition conditions. In these studies, the compressive stress has been reported to be typical for sputtered thin films, and it has been related to the atomic peening mechanism inherent to the sputtering process [11], [26], [27], whereas the tensile stress is thought to arise from other defects [11]. It is worthwhile to mention that films grown by e-beam display a typical tensile stress, which can be reduced by increasing the deposition temperature [12], [16].

The surface morphology of the films does not seem to display a clear dependence on $V_{c}$ in the studied range. All layers are very flat, with typical values for integrated surface roughness (rms) over $1 \mu \mathrm{m}^{2}$ ranging from 0.13 to $0.32 \mathrm{~nm}$. The low rms might indicate, as further evidenced by TEM experiments, that the films present a compact microstructure, which has been commonly associated with the compressive stress [28].

Planar TEM and SEM images reveal that the films are quite granular (Fig. 4). Transversal TEM images in similar films have shown that they are made of tightly packed columns with height equal to the thickness of the films [19]. The columnar growth of Mo is typical of metal films grown at temperatures much lower than their melting temperature $\left(T_{\text {melting }} / T_{\text {substrate }} \gg\right.$ $1)$; indeed, similar morphologies have been reported for Mo films deposited by either sputtering or e-beam, with grain sizes ranging from 3 to $5 \mathrm{~nm}$ (sputtering [11], [26]) to $40 \mathrm{~nm}$ (e-beam [2], [3], [5]).

As shown in Fig. 4, there is a considerable distribution in the lateral size and shape of the columns within each film. Most of the grains are of about $\sim 20 \mathrm{~nm}$, but there are larger grains, on the order of $35-40 \mathrm{~nm}$, as well as a few even larger $(\sim 70 \mathrm{~nm})$. The boundaries of the grains are some times diffuse or difficult to discern; it might very well be that these larger grains are in fact an agglomeration of smaller grains. No clear variation of

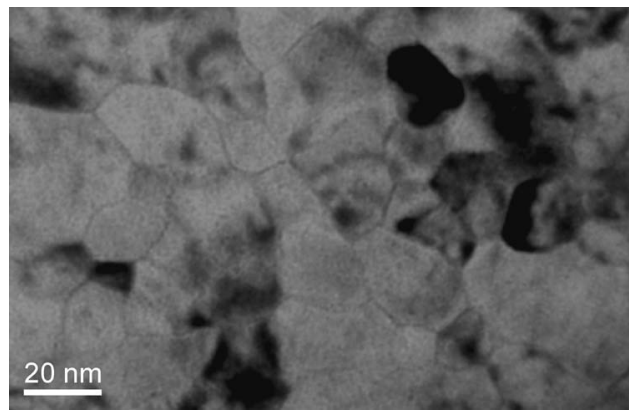

Fig. 4. Planar TEM image of the film deposited at $V_{c}=248 \mathrm{~V}$. A large dispersion of the grain sizes and shapes is visible for all films.
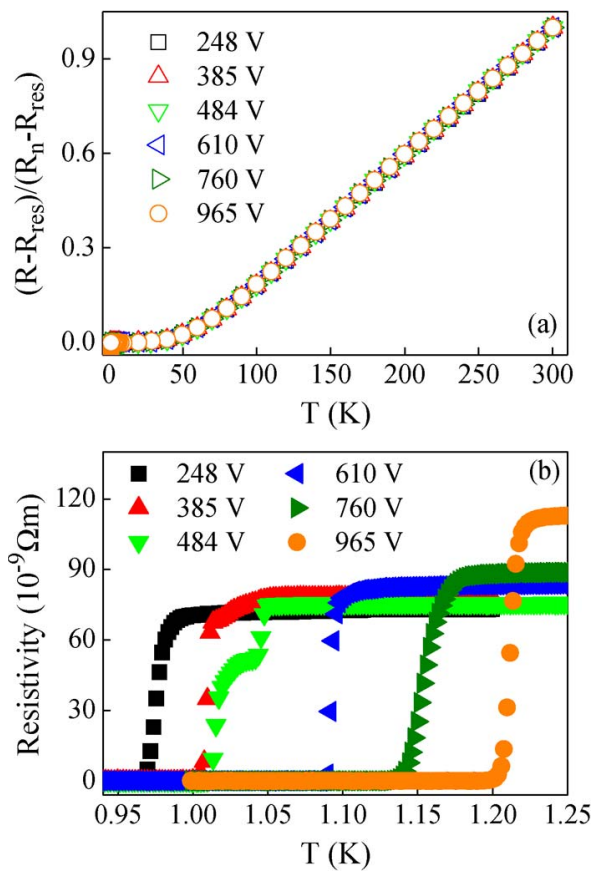

Fig. 5. (a) Temperature dependence of the normal-state resistivity for all the films. Data have been plotted as $R(T)-R_{\text {res, }}$ normalized to its value at $300 \mathrm{~K}$, to emphasize that the temperature-dependent part of the resistivity is identical for the six films: All of them display the same slope, i.e., $0.00409 \pm$ 0.00001 , being the error bar of each fit smaller than $10^{-5}$. (b) Superconducting transitions of the films.

the grain-size distribution as a function of $V_{c}$ has been observed; this is likely due to the large dispersion. ${ }^{1}$

\section{B. Normal-State Resistivity}

The temperature dependence of resistance $R(T)$ for the studied films is depicted in Fig. 5. The values of the normalstate resistivity at $300 \mathrm{~K}$, i.e., $\rho_{n}$, and the residual resistivity at $4.2 \mathrm{~K}$, i.e., $\rho_{\text {res }}$, are given in Table $\mathrm{I}$, together with the superconducting critical temperature $T_{c}$. The values of $\rho_{n}$ agree quite well with those typically reported for Mo thin films [5],

\footnotetext{
${ }^{1}$ Several authors have tried to extract grain sizes from the widths of XRD peaks. However, this is difficult in stressed textured columnar films for two reasons: First, in the presence of stress, the broadening of the peak caused by microstresses must be taken into account, and usually, it is difficult to subtract. Second, for highly oriented films, the use of the main peak-as usually reported-can only provide the grain size along the growth direction, i.e., the column height in this case.
} 
TABLE I

Electric And Superconducting PARAmeters of THE Films

\begin{tabular}{|c|c|c|c|c|c|}
\hline $\mathrm{W}(\mathrm{W})$ & $\mathrm{V}_{\mathrm{c}}(\mathrm{V})$ & $\mathrm{T}_{\mathrm{c}}(\mathrm{mK})$ & RRR & $\rho_{\mathrm{n}}(\mu \Omega \mathrm{cm})$ & $\rho_{\text {res }}(\mu \Omega \mathrm{cm})$ \\
\hline 25 & 248 & 975 & 2.04 & 14.91 & 7.31 \\
\hline 50 & 385 & 1009 & 1.98 & 15.47 & 7.83 \\
\hline 75 & 484 & 1017 & 1.96 & 14.80 & 7.55 \\
\hline 105 & 610 & 1091 & 1.83 & 15.38 & 8.41 \\
\hline 155 & 760 & 1150 & 1.70 & 15.21 & 8.93 \\
\hline 213 & 965 & 1209 & 1.64 & 18.60 & 11.31 \\
\hline
\end{tabular}

Superconducting critical temperature $T_{c}$, residual resistance ratio $R R R$ and resistivity values at $300 \mathrm{~K}\left(\rho_{\mathrm{n}}\right)$ and $4.2 \mathrm{~K}\left(\rho_{\text {res }}\right)$, for films grown at the given $\mathrm{RF}$ power $\mathrm{W}$ and bias voltage $\mathrm{V}_{\mathrm{c}}$
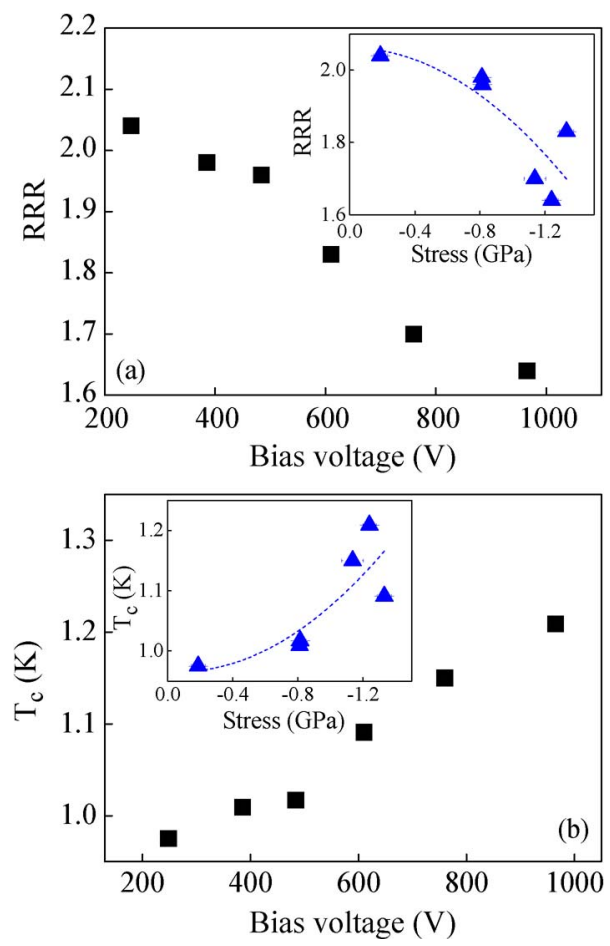

Fig. 6. (a) RRR as a function of the bias voltage $V_{c}$. (Inset) RRR as a function of the measured residual stress of the films. (b) Superconducting critical temperature $T_{c}$ as a function of the bias voltage. (Inset) $T_{c}$ as a function of the measured residual stress.

[6], [15]-[19] and are up to a factor of 4 higher than those for bulk Mo, i.e., $\rho_{n}^{\text {bulk }}=5.3 \mu \Omega \cdot \mathrm{cm}$. The residual resistance ratio $(\mathrm{RRR})$ value $\left(\mathrm{RRR}=\rho_{n} / \rho_{\text {res }}\right)$ decreases with raising $V_{c}$, from 2.04 to 1.64 for films deposited at 248 and $965 \mathrm{~V}$, respectively [Fig. 6(a)]. These values are very much similar to those usually found for Mo thin films deposited by sputtering and e-beam but are substantially lower than those of high-purity Mo single crystals. The high $\rho_{n}$ and low RRR values of granular metal thin films are usually ascribed to enhanced scattering occurring at grain boundaries [2], [3], [18], [29], [30]. This fact is further analyzed in the succeeding paragraphs.

Both $\rho_{n}$ and $\rho_{\text {res }}$ increase as a function of $V_{c}$, but RRR displays a steady decrease with increasing $V_{c}$ [Fig. 6(a)]. A closer analysis of the data reveals that the linear temperature dependence of $R(T)$ above the residual value $R_{\text {res }}$ is independent of $V_{c}$. This may clearly be appreciated in Fig. 5(a), where the difference $R(T)-R_{\text {res }}$, which is normalized to the $T=$ $300 \mathrm{~K}$ value (to avoid errors associated with the calculation of resistivity using geometry factors), has been plotted as a function of temperature. In this figure, it is observed that the data for all films collapse onto a unique straight line. These data evidence that the temperature-dependent part of the resistivity is not altered by changing $V_{c}$, whereas the residual resistance increases with $V_{c}$ and is responsible for the behavior observed in Fig. 6(a).

As it is well known, the resistivity of a metal has two components: 1) electron scattering with phonons and 2) electron scattering with lattice imperfections. The first component, which is typically proportional to temperature, mainly depends on the phonon concentration; the second component, which is usually identified as $\rho_{\text {res }}$, is the one that dominates the lowtemperature behavior. Fig. 5(a) reveals that, in our films, the two resistivity components are independent, i.e., electron scattering with lattice defects does not depend on $T$, whereas electron scattering with phonons is independent of the lattice defects. This is known as the Matthiessen's rule. Thus, this analysis evidences that, as previously stated, changes in $V_{c}$ only affect $\rho_{\text {res }}$, i.e., electron scattering with lattice defects is enhanced with $V_{c}$, and this can only happen if the density of lattice defects increases. We examine in the following the possible origins for this effect.

Sputtered films can contain a number of impurities, which may be incorporated during deposition either from the residual gas or from the sputtering gas (Ar). In the first case, the presence of impurities should be enhanced at lower deposition rates, and therefore, an increase of $\rho_{\text {res }}$ would be expected with diminishing $V_{c}$. Nevertheless, this would be in opposition to the behavior observed in Fig. 6(a). On the other hand, the incorporation of Ar should increase when raising the energy of the atoms ejected from the target (i.e., increasing $V_{c}$ ), which would account for the tendency displayed by $\rho_{\text {res }}\left(V_{c}\right)$.

No impurity traces have been detected by RBS, with the exception of an atomic percentage of argon $(>2 \%)$ [20], which appears to become higher with increasing $V_{c}$. However, the density of the incorporated Ar atoms is very small that they cannot be considered to be the main factor that is responsible for the measured changes in $\rho_{\text {res }}(55 \%$ of increase between the films deposited at 248 and $965 \mathrm{~V}$ ). Indeed, a typical resistivity enhancement on the order of $1 \mu \Omega \cdot \mathrm{cm}$ per atomic percentage of impurities has been reported for many metals [31], whereas the changes in resistivity depicted in Table I for our particular films are several times larger than this.

The second possible source of increased static electron scattering is grain boundaries. In fact, the enhanced resistivity of granular metal thin films is usually accounted for by this effect. The Mayadas-Shatzkes expression [29] allows one to estimate the resistivity $\rho_{n}^{\text {film }}$ of granular disordered films from the bulk value $\rho_{n}^{\text {bulk }}$, i.e.,

$$
\rho_{n}^{\text {bulk }}=\rho_{n}^{\text {film }} 3\left[1 / 3-\alpha / 2+\alpha^{2}-\alpha^{3} \ln (1+1 / \alpha)\right] .
$$

The parameter $\alpha$ is defined as $\alpha \equiv R /(1-R) l_{o} / D$, where $D$ is the average grain size in the film, $R$ is a factor corresponding to the fraction of electrons specularly reflected at the boundary, 
and $l_{o}$ is the electron mean free path of the metal. Equation (1) reveals that, when $D$ approaches $l_{o}$, the resistivity of the film is very sensitive to $D$ and can suffer a significant enhancement. This is indeed the case in our films: For Mo, $l_{o}$ is $40 \mathrm{~nm}$ [30], whereas the lateral grain size of the columns is on this order or smaller. Therefore, grain boundary scattering is a plausible explanation for the observed increase of $\rho_{\text {res }}$. This argument may also be coherent if it is considered that an increase in the growth rate should reduce the grain size and therefore make grain boundary scattering more relevant. Unfortunately, because of the observed large dispersion in grain sizes, such a hypothesis cannot directly be corroborated from our experimental data. Nevertheless, some estimates can be done to deduce if variations in $D$ within the observed dispersion could account for the changes measured in the resistivity. Using $D=25 \mathrm{~nm}$ and $\rho$ corresponding to the film grown at the lowest $V_{c}$, (1) allows one to estimate $R \sim 0.4$, which is a rather acceptable value [2]. Assuming that $R$ is constant, the grain size that would give rise to the measured resistivity for the film grown at the highest $V_{c}$ could be explained by a grain size of $D=20 \mathrm{~nm}$. These results prove that $\rho$ is very sensitive to small changes - within our experimental dispersion — in the grain size $D$. Because of this reason, grain boundary scattering can be assumed to be a good candidate to account for the behavior observed in Fig. 6(a).

Finally, other lattice defects can be present in the films, and their density should increase with increasing stress. In fact, the resistivity $R(T)$ of a metal is quite insensitive to stress because it produces tiny changes of the lattice parameter. However, stress may have indirect effects on $\rho_{\text {res }}$ through the generation of structural defects and morphology changes (including a reduction of the grain size). These could explain the behavior depicted in the inset of Fig. 6(a).

\section{Superconducting Critical Temperature}

We now turn to the superconducting critical temperature $T_{c}$. Several reports have emphasized the relationship between $T_{c}$ and stress in Mo films. For films deposited by e-beam, $T_{c}$ and RRR rise as tensile stress is suppressed [5], [15]-[18]. A few reports focused on dc magnetron-sputtered films report that the best $T_{c}(\sim 1-1.4 \mathrm{~K})$ and reproducibility are obtained for slightly compressed films ( $\sigma \sim-100 \mathrm{MPa})$ [6], [17]. Nonetheless, to our knowledge, the relationship between $T_{c}$, RRR, and stress for Mo thin films deposited by sputtering has not yet been clearly settled.

As shown in Table I and Fig. 6(b), $T_{c}$ values higher than those corresponding to bulk Mo $\left(T_{c}^{\mathrm{bulk}}=915 \mathrm{mK}\right)$ have been measured for all films. In general, a gradual enhancement of $T_{c}$ is observed when raising $V_{c}$. From the dependences of stress and RRR with $V_{c}$ [Figs. 3 and 6(a)], it follows that the enhancement of $T_{c}$ may also be linked to an increase of the residual stress [inset of Fig. 6(b)] or, equivalently, to a reduction of RRR (Fig. 7).

All the films that we have been deposited and analyzed display the same dependence of $T_{c}(\mathrm{RRR})$ depicted in Fig. 7 [19]. Thus, this dependence is highly reproducible. However, such dependence is found to be quite anomalous among clas-

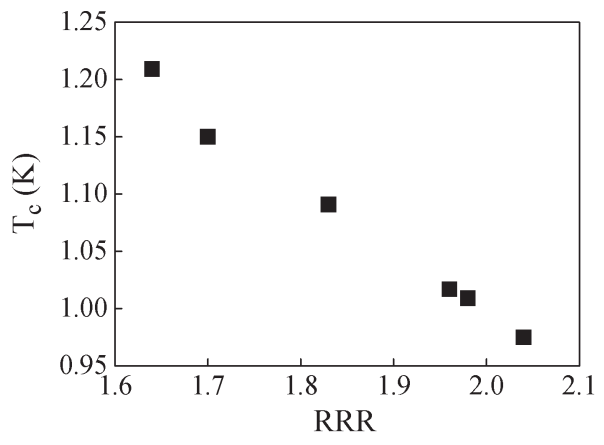

Fig. 7. Superconducting critical temperature $T_{c}$ as a function of the RRR of the films. A highly reproducible [19] linear dependence is observed.

sical metal superconductors. Indeed, the $T_{c}$ of most of them (e.g., $\mathrm{Nb}, \mathrm{Ta}, \mathrm{V}$, and $\mathrm{Al}$ ) gets lower when increasing the residual resistance (mainly because of impurity incorporation), which has been explained by a reduction of the mean free path [32]-[34]. It should be stated, however, that a rise of $T_{c}$ with increasing $\rho_{\text {res }}$ is possible, depending on the origin or type of defects dominating $\rho_{\text {res }}$. Indeed, within the Bardeen-Cooper-Schrieffer theory, changes of $T_{c}$ - either enhancement or suppression-are the result of changes in the density of states or in the electron-phonon coupling; on the other hand, changes in electron scattering without substantially modifying the electron or phonon spectra should have tiny effects on $T_{c}$. To understand the mechanism responsible for these $T_{c}$ changes, we will focus on the different factors that we have shown to change with $V_{c}$, as well as on their correlation with resistivity.

The measured $T_{c}$ values, as stated above, are larger than the bulk $T_{c}$; even more, an increment of $\rho_{\text {res }}$ of $55 \%$ results in a substantial increase of $T_{c}$ of $25 \%$. Both RRR (or $1 / \rho_{\text {res }}$ ) and $T_{c}$ display a clear linear dependence on $V_{c}$, whereas the dependence on stress exists but is less neat [inset of Fig. 6(b)]. In addition, as discussed above, the increase of $\rho_{\text {res }}$ with $V_{c}$ can be understood by an enhancement of electron scattering by grain boundaries or other stress-generated defects.

The traces of Ar contained in the films are unlikely to explain the significant rise of $T_{c}$ with $V_{c}$; in fact, D'yakov and Shvets found an enhancement of $T_{c}$ above the bulk value for Mo single crystals with impurities, but $T_{c}$ changed 29 by $\mathrm{mK}$, whereas $\rho_{\text {res }}$ changed by a factor of 35 [35].

Stress can produce large changes in $T_{c}$ since it may change both the electronic structure and the phonon spectrum of a material [34], [36]-[39]. McMillan [40] showed that metals with weak electron-phonon coupling and low $T_{c}$, such as Mo, could display large increases in $T_{c}$ upon decreasing the average phonon frequency. In fact, Kar'kin et al. [41] have reported that superconducting two-element compounds with Mo or $\mathrm{Nb}$ behave in a different way under pressure. While for $\mathrm{Nb}$ compounds $T_{c}$ clearly increases with RRR, Mo compounds display a less clear trend, with a tendency of $T_{c}$ to increase for decreasing RRR; the authors have argued that this different behavior must be related to distinct changes of the electron and phonon spectra of $\mathrm{Nb}$ and Mo associated with microstresses, which could also explain the anomalous $T_{c}(\mathrm{RRR})$ in our Mo films. 
Finally, we must discuss whether the granular character of the films, which we have considered as a very likely cause for the changes of $\rho_{\text {res }}$, may be responsible for the changes in $T_{c}$. It has been argued [42] that, in granular films, the average ionic vibration amplitude increases because the ionic restoring forces are weaker at the grain surfaces, due to reduced symmetry. This effect implies an increase of the phonon amplitude and a reduction of their average frequency, causing an increase of the electron-phonon coupling and, therefore, an increase of $T_{c}$.

In summary, both the enhancement of $T_{c}$ in comparison with bulk and the $T_{c}(\mathrm{RRR})$ dependence could be explained by changes in the electronic density of states caused by compressive stress, or by an increase of the electron-phonon coupling associated with stress or with the progressively reduced grain size.

\section{CONCLUSION}

The effects of morphology and residual stress on the resistivity and superconducting critical temperature of Mo thin films deposited by RF magnetron sputtering at room temperature and at different dc bias $V_{c}$ have been studied.

The residual resistance and critical temperature have been found to increase with $V_{c}$. The measured changes in the residual resistance are associated with enhanced electron scattering due to grain boundaries and other lattice defects, which are generated by increased compressive stress as $V_{c}$ rises.

The enhancement of $T_{c}$ accompanying the decrease of RRR (increase of the residual resistance) is extremely reproducible although quite anomalous among metal element superconductors. We have shown that this behavior can be explained by stress and morphology changes and must be related to the specific electron and phonon spectra of Mo.

\section{ACKNOWLEDGMENT}

R. González-Arrabal and J. Sesé would like to thank MEC for their Juan de la Cierva and Ramon y Cajal contracts, respectively. O. Gil and M. Parra-Borderías would like to thank MCINN for their fellowship.

\section{REFERENCES}

[1] Y. G. Shen, "Effect of deposition conditions on mechanical stresses and microstructure of sputter-deposited molybdenum and reactively sputterdeposited molybdenum nitride films," Mater. Sci. Eng. A, vol. 359, no. 1, pp. $158-167$, Oct. 2003.

[2] H. Oikawa, "Electrical resistivity of vacuum-deposited molybdenum films," J. Vac. Sci. Technol., vol. 15, no. 3, pp. 1117-1122, May 1978.

[3] H. Oikawa and Y. Nakajima, "Effect of heat treatment after deposition on internal stress in molybdenum films on substrates," J. Vac. Sci. Technol., vol. 14, no. 5, pp. 1153-1156, Sep. 1977.

[4] H. Khatri and S. Marsillac, "Effect of deposition parameters on r.f. sputtered molybdenum thin films," J. Phys. Condens. Mater, vol. 20, no. 5, p. 055206 , Jan. 2008.

[5] N. Tralshawala, R. P. Brekosky, M. J. Li, E. Figueroa-Feliciano, F. M. Finkbeiner, M. A. Lindeman, C. M. Stahle, and C. K. Stahle, "Fabrication of Mo/Au transition-edge sensors for X-ray spectrometry," IEEE Trans. Appl. Supercond., vol. 11, no. 1, pp. 755-758, Mar. 2001.

[6] G. C. Hilton, J. M. Martinis, K. D. Irwin, N. F. Bergren, D. A. Wollman, M. E. Huber, S. Deiker, and S. W. Nam, "Microfabricated transition-edge X-ray detectors," IEEE Trans. Appl. Supercond., vol. 11, no. 1, pp. 739742, Mar. 2001
[7] A. G. Blachman, "Stress and resistivity control in sputtered molybdenum films and comparison with sputtered gold," Metallurg. Mater. Trans., vol. 2, no. 3, pp. 699-709, Mar. 1971.

[8] S. G. Malhotra, Z. U. Rek, S. M. Yalisove, and J. C. Bilello, "Depth dependence of residual strains in polycrystalline Mo thin films using highresolution X-ray diffraction," J. Appl. Phys., vol. 79, no. 9, pp. 6872-6879, May 1996.

[9] D. W. Hoffman and C. M. Kukla, "Determination of film stresses during sputter deposition using an in situ probe," J. Vac. Sci. Technol. A, Vac. Surf. Films, vol. 3, no. 6, pp. 2600-2604, Nov. 1985.

[10] A. Debelle, G. Abadias, A. Michel, and C. Jaouen, "Stress field in sputtered thin films: Ion irradiation as a tool to induce relaxation and investigate the origin of growth stress," Appl. Phys. Lett., vol. 84, no. 24, pp. 5034-5036, May 2004.

[11] T. J. Vink, M. A. J. Somers, J. L. C. Daams, and A. G. Dirks, "Stress, strain, and microstructure of sputter-deposited Mo thin films," J. Appl. Phys., vol. 70, no. 8, pp. 4301-4308, Oct. 1991.

[12] R. A. Holmwood and R. Glang, "Vacuum deposited molybdenum films," J. Electrochem. Soc., vol. 112, no. 8, pp. 827-831, Aug. 1965.

[13] M. Guilloux-Viry, A. Perrin, J. Padiou, M. Sergent, and C. Rossel, "Epitaxially grown molybdenum thin films deposited by laser ablation on (100) MgO substrates," Thin Solid Films, vol. 280, no. 1/2, pp. 76-82, Jul. 1996.

[14] C. Dicov, M. Marinov, H. Maciel, K. Grigorov, I. Nedkov, and G. Beshkov, "Properties of $\mathrm{Cr}$ and Mo thin films deposited by RF sputtering," J. Optoelectron. Adv. Mater, vol. 7, no. 1, pp. 385-387, 2005.

[15] F. M. Finkbeiner, T. C. Chen, S. Aslam, E. Figueroa-Feliciano, R. L. Kelley, M. Li, D. B. Mott, C. K. Stahle, and C. M. Stahle, "Fabrication of superconducting bilayer transition edge thermometers and their application for spaceborne X-ray microcalorimetry," IEEE Trans. Appl. Supercond., vol. 9, no. 2, pp. 2940-2942, Jun. 1999.

[16] J. A. Chervenak, F. M. Finkbeiner, T. R. Stevenson, D. J. Talley, R. P. Brekosky, S. R. Bandler, E. Figueroa-Feliciano, M. A. Lindeman, R. L. Kelley, T. Saab, and C. K. Stahle, "Fabrication of transition edge sensor X-ray microcalorimeters for constellation-X," Nucl. Instrum. Methods Phys. Res. A, Accel. Spectrom. Detect. Assoc. Equip., vol. 520, no. 1-3, pp. 460-462, Mar. 2004.

[17] J. A. Chervenak, F. M. Finkbeiner, S. R. Bandler, R. Brekosky, A. D. Brown, N. Iyomoto, R. L. Kelley, C. A. Kilbourne, F. S. Porter, J. Sadleir, and S. Smith, "Materials development for auxiliary components for large compact Mo/Au TES arrays," J. Low Temp. Phys., vol. 151, no. $1 / 2$, pp. 255-260, Apr. 2008.

[18] E. Monticone, M. Rajteri, C. Portesi, C. Gandini, S. Bodoardo, and G. B. Picotto, "Structural and electrical characterisation of Mo films for transition-edge sensors," Phys. C, vol. 372-376, pp. 440-443, Aug. 2002.

[19] L. Fàbrega, I. Fernandez-Martinez, Ó. Gil, M. Parra-Borderias, A. Camon, J.-L. Costa-Kramer, R. Gonzalez-Arrabal, J. Sese, F. Briones, J. Santiso, and F. Peiro, "Mo-based proximity bilayers for TES: Microstructure and properties," IEEE Trans. Appl. Supercond., vol. 19, no. 3, pp. 460-464, Jun. 2009.

[20] R. González-Arrabal, A. Camón, M. Parra-Borderías, L. Fabrega, J. Anguita, J. Sesé, and F. Briones, "Mo/Au bilayers deposited by sputtering at room temperature for transition edge sensors fabrication," J. Low Temp. Phys., vol. 151, no. 1/2, pp. 239-244, Apr. 2008.

[21] D. J. W. Mous, A. Gottdang, and R. G. Haitsma, "The novel HVEE 5MV Tandetron," Nucl. Instrum. Methods Phys. Res. B, Beam Interact. Mater. At., vol. 190, no. 1-4, pp. 177-184, 2002.

[22] D. J. W. Mous, A. Gottdang, R. G. Haitsma, G. Garcia Lopez, A. ClimentFont, F. Agulló-López, and D. O. Boerma, "Performance and applications of the first HVE 5 MV Tandetron at the University of Madrid," in Proc. 17th Int. Conf. Appl. Accel. Res. Ind., L. Duggan and I. L. Morgan, Eds., College Park, MD, 2003, vol. CP680, pp. 999-2002.

[23] M. Mayer, SIMNRA. Munich, Germany: Max Plank Institut für Plasmaphysik.

[24] B. D. Cullity and S. R. Stock, Elements of X-ray Diffraction. Englewood Cliffs, NJ: Prentice-Hall, 2001.

[25] A. Bensaoula, J. C. Wolfe, A. Ignatiev, F-O. Fong, and T-S. Leung, "Direct-current-magnetron deposition of molybdenum and tungsten with rf-substrate bias," J. Vac. Sci. Technol. A, Vac. Surf. Films, vol. 2, no. 2, pp. 389-392, Apr. 1984.

[26] N. Y. Fogel, O. A. Koretskaya, A. S. Pokhila, V. G. Cherkasova, E. I. Buchstab, and S. A. Yulin, "Mo films and Mo/Si superlattices: Comparison of superconducting and kinetic parameters," J. Low Temp. Phys., vol. 22, no. 4, pp. 277-280, Apr. 1996.

[27] J. A. Thornton and D. W. Hoffman, "Stress-related effects in thin films," Thin Solid Films, vol. 117, no. 1, pp. 5-31, Apr. 1989. 
[28] J. A. Thornton and D. W. Hoffman, "Internal stresses in titanium, nickel, molybdenum, and tantalum films deposited by cylindrical magnetron sputtering," J. Vac. Sci. Technol., vol. 14, pp. 164-168, 1977.

[29] P. Wiâmann, Electrical Resistivity of Metals and Alloys. Berlin, Germany: Springer-Verlag, 1987, p. 25.

[30] C. Kittel, Introduction to Solid State Physics. New York: Wiley, 1986, p. 145.

[31] E. Fawcett and D. Griffiths, "The Fermi surface areas of chromium, molybdenum and tungsten," J. Phys. Chem. Solids, vol. 23, no. 11, pp. 1631-1635, Nov. 1962.

[32] J. R. Rairden and C. A. Neugebauer, "Critical temperature of niobium and tantalum films," Proc. IEEE, vol. 52, no. 10, pp. 1234-1238, Oct. 1964.

[33] G. Boato, G. Gallinaro, and C. Rizzuto, "Superconductive transition of aluminium containing transition metal impurities," Phys. Lett., vol. 5, no. 1, pp. 20-21, Jun. 1963

[34] D. P. Seraphim, D. T. Novick, and J. I. Budnick, "The effects of imperfections on the superconducting critical temperature of tantalum," Acta Metallurgica, vol. 9, pp. 446-453, 1961.

[35] I. G. D'yakov and A. D. Shvets, "Investigation of superconducting properties of molybdenum," Sov. Phys. JETP, vol. 22, pp. 759-761, Apr. 1966.

[36] P. M. Hall, "Effect of stress on the superconducting transition temperature of thin films of tin," J. Appl. Phys., vol. 36, no. 8, pp. 2471-2475, Aug. 1965.

[37] V. M. Kuz'menko and T. P. Chernyaeva, "Influence of internal stresses on the superconductivity of nanocrystalline vanadium," J. Low Temp. Phys., vol. 31, no. 2, pp. 111-115, Feb. 2005.

[38] J. J. Cuomo, "Modification of niobium film stress by low-energy ion bombardment during deposition," J. Vac. Sci. Technol., vol. 20, no. 3, pp. 349-354, Mar. 1982.

[39] V. I. Dotsenko, I. F. Kislyak, V. T. Petrenko, M. A. Tikhonovsky, A. M. Shkilko, L. N. Zagoruiko, and L. M. Rogozyanskaya, "Static stress effects on superconducting composites-Part II. Effect of uniaxial tensile and compressive stresses on the critical temperatures of $\mathrm{Cu}-\mathrm{Nb}, \mathrm{Cu}-\mathrm{NbTi}$, and bronze-Nb ${ }_{3}$ Sn composites," Cryogenics, vol. 41, no. 4, pp. 225-230, Apr. 2001

[40] W. L. McMillan, "Transition temperature of strong-coupled superconductors," Phys. Rev., vol. 167, no. 2, pp. 331-344, Mar. 1967.

[41] A. Y. Kar'kin, V. P. Piliugin, V. I. Voronin, R. I. Kuznetsov, and B. N. Goshchitskii, "Superconductivity and the structure of materials based on niobium and molybdenum, deformed under pressure," Phys. Met. Metall., vol. 66, pp. 102-109, Jul. 1988.

[42] J. W. Garland, K. H. Bennemann, and F. M. Mueller, "Effect of lattice disorder on the superconducting transition temperature," Phys. Rev. Lett., vol. 21 , no. 18 , pp. $1315-1319$, Oct. 1968.
Lourdes Fàbrega, biography not available at the time of publication.

Iván Fernández-Martínez, biography not available at the time of publication.

María Parra-Borderías, biography not available at the time of publication.

Óscar Gil, biography not available at the time of publication.

Agustín Camón, biography not available at the time of publication.

Raquel González-Arrabal, biography not available at the time of publication.

Javier Sesé, biography not available at the time of publication.

José Santiso, biography not available at the time of publication.

José-Luis Costa-Krämer, biography not available at the time of publication.

Fernando Briones, biography not available at the time of publication. 